common to three of them will be represented by the vertex in which three planes intersect. This gives a symmetrical representation of the relations among the eight subclasses $x y z$, $x y z^{\prime}, \ldots$

For practical use in the solution of logical problems, a convenient diagram is obtained by drawing a plane projection of a cube and marking each face with its corresponding class symbol. At each vertex may be left a circular space which, in any given case, may receive a distinguishing mark in accordance with the import of a particular proposition. Such a diagram is represented in Fig. 1.

A symmetrical representation for the case of $n$ primary class terms would require a figure in $n$ dimensions. But a plane diagram for practical use may be made by repeating the diagram for the case of three terms. For example, the case of six primary terms may be represented by eight cubes, each placed at a vertex of a larger cube. Thus the projection shown in Fig. 1 might be drawn on a large scale, and a similar projection drawn in each of the circular spaces; each of these smaller figures representing the relations of three primary terms $u, v$, $w$ and their negatives.

For practical use a permanent diagram may be drawn upon a slate or blackboard, so that the marks used in particular problems may readily be erased.

\title{
HEINRICH MASCHKE: HIS LIFE AND WORK.
}

Heinrich Maschke was born at Breslau in Germany on October 24, 1853. He received his early education at the Maria-Magdalenen-Gymnasium of his native town, where his exceptional mathematical talent soon attracted the attention of his teachers. In 1872 he graduated from the gymnasium and entered the University of Heidelberg as a student of mathematics, the science which he had selected for his life study. After several semesters at Heidelberg, where he was initiated by Königsberger into the mysteries of infinitesimal calculus, and at Breslau, where he served his year as "Einjährig-Freiwilliger" in the Prussian army, he went for three years to to Berlin, attracted by the famous triad Weierstrass, Kummer, and Kronecker, of whom Kummer seems to have had the most lasting influence upon his mathematical development. 
In 1878 he passed with high distinction the government examination (Staatsexamen) in Berlin and in 1880 the doctor's examination in Göttingen with a thesis "Ueber ein dreifach orthogonales Flächensystem, gebildet aus Flächen dritter Ordnung."

Soon afterwards he accepted a position as teacher of mathematics in the Luisenstädtische Gymnasium in Berlin. But though he was a most successful teacher, he soon felt that the work in the gymnasium, which consisted in teaching, for about twenty hours a week, arithmetic and the first elements of algebra and geometry, could not permanently satisfy him. As time went on, this feeling increased, especially after his return to the gymnasium, in 1887, from a year's leave of absence spent at the University of Göttingen under Professor Felix Klein. But there was no hope that he could ever cross the almost insurmountable barriers between the gymnasium and the university, and only a radical step could bring the deliverance from an occupation which had more and more become an irksome burden.

Shortly before, two of his nearest friends had, under similar circumstances, emigrated to the United States and soon found suitable positions. Their success and urgent persuasion determined Maschke to throw up his position and follow their example. But as he did not wish to rely entirely upon the doubtful chances of finding a university position, he employed his leisure hours during the last year of his stay at the gymnasium in studying electrotechnics at the Polytechnicum in Charlottenburg. In July, 1890, he left the gymnasium for good, spent several months in practical electrical work in the Berliner Allgemeine Electricitätsgesellschaft, and completed his electrotechnical studies during the winter semester 1890/91 at the Polytechnicum in Darmstadt under Professor Kittler.

Thus prepared he landed in New York April 1, 1891. Soon afterwards he found a position as electrician in the Weston Electric Instrument Company of Newark, N. J., and one year later he accepted a call as assistant professor of mathematics in the newly founded University of Chicago.

Here at last he found the place best suited to his abilities, where he could freely develop his powers in a sphere of farreaching usefulness, $*$ and he has always gratefully acknowledged his deep indebtedness to this hospitable country of great

* More than seventy of the present members of the American Mathematical Society have, at one time or another, attended Maschke's courses. 
opportunities which has made it possible for him to live out his own life.

With love and enthusiasm, and with the greatest success, he devoted himself to his new duties. His rare pedagogical talent, aided by his long experience in the gymnasium, his genuine scholarly spirit, a strong artistic sense which gave a special charm to his lectures, together with his fine personal qualities combined to make him an ideal teacher. His courses covered a wide range of different subjects : synthetic and analytic geometry, algebraic curves and differential geometry; function theory, elliptic functions, linear differential equations; theory of equations, invariants, groups ; analytical mechanics, potential, electricity, - are only a partial list of the subjects on which he lectured in the course of the nearly sixteen years which he was connected with the University of Chicago, first as assistant professor, then as associate, and finally as full professor.

As an investigator Maschke has made his mark in two different branches of mathematics : in the theory of finite groups of linear substitutions and in the theory of quadratic differential quantics. A detailed appreciation of his contributions will be given below; they are all distinguished by a peculiar beauty and elegance, emanating from that same artistic sense which made his lectures so attractive.

Through his teaching, his publications, and his activity in the American Mathematical Society, especially the Chicago Section, Maschke has had a considerable share in the modern movement in mathematics in this country. He was a member of the Council in 1902-1905, and Vice-President of the Society during 1907. In the same connection his address "On present problems of algebra and analysis" at the St. Louis international congress of 1904 should be mentioned.

Many of the readers of these lines have known him personally; they all remember his sunny disposition, his genuine kindness and modesty, qualities which quickly won him the hearts of all with whom he came in contact. But the peculiar charm of his personality lay in its harmoniousness as a whole, in the beautiful balance of his mental and moral faculties which was the source of his quiet strength and manly dignity.

Maschke had always enjoyed excellent health, and there was every prospect that he would live to old age. But it was not to be. Towards the end of 1907 symptoms of an internal disorder began to show themselves; an examination 
which took place in the last week of February, 1908, revealed the necessity of an immediate operation, to which he succumbed on March 1.

Maschke's career as an investigator began in 1886 at Göttingen under the inspiring influence of Professor Felix Klein. Only two years before, Klein's Vorlesungen über das Ikosaeder had been published, and in the meantime Klein * had developed the extension of his solution of the general equation of the fifth degree, in which the solution of an algebraic equation of a given group is considered as a special case of the socalled form problem for a finite group of linear substitutions, $i$. e., the problem of determining the values of the independent variables of the substitution group when the values of the invariant forms of the group are given. Hence the great interest which attached to the problem : to determine the complete system of invariant forms for a given finite group of linear substitutions. For the binary groups this problem had been solved by Schwarz, Klein, and Gordan, for the ternary $G_{168}$ by Klein ; it was still unsolved for the remaining ternary groups and for the quaternary groups then known. The most interesting of the latter had been derived by Klein, partly from line geometry, partly from the transformation theory of hyperelliptic functions, viz., 1) the so-called group of the Borchardt moduli, a group of 64.720 substitutions, isomorphic with the alternating group of six letters ; 2 ) the group of Klein's hyperelliptic moduli $Z_{\alpha \beta}$ for the transformation of the third degree, a group of 51840 substitutions, isomorphic with the group of the equation of the 27 straight lines on the general cubic surface ; 3) a group of $2 \cdot 7 ! / 2$ substitutions, derived by Klein from the consideration of "supernumerary" line coordinates and isomorphic with the alternating group of seven letters.

The first problem which Maschke took up, while still at Göttingen, was the determination of the complete system of invariant forms for the group of the Borchardt moduli. $\dagger$ Considering the high order of the group, the problem seems at first sight one of appalling difficulty. But it was precisely this character of the problem which attracted Maschke; he was a very adroit and indefatigable calculator and he delighted in

* Compare Wiman's article in the Encyklopädie, I B 3 f.

$\dagger$ "Ueber die quaternäre endliche, lineare Substitutionsgruppe der Borchardtschen Moduln," Göttinger Nachrichten, 1887, p. 421, and Math. Annalen, vol. 30, p. 496 (June, 1887). 
attacking a problem of apparently insurmountable difficulty, reducing first by preliminary ingenious devices the amount of necessary computation to a minimum and taking finally the rest by sheer force. After having derived a system of generating substitutions for his group $G$ in a particularly simple and handy form, he first determines without much difficulty the invariant forms for a certain invariant subgroup of $G$ of order 64. They are five in number and of degree four, and they are linearly substituted among themselves under the application of the main group $G$. Next he introduces a linear combination $\Phi$ of these five forms which is six-valued under a subgroup of $G$ of index 2 , and out of these six values of $\Phi$ the invariant forms of $G$ are finally built up.

Maschke made an interesting application of his results to the solution of the general equation of the sixth degree.* By means of a Tschirnhausen transformation he identifies the general equation of the sixth degree with the resolvent equation satisfied by the six values of the function $\Phi$ mentioned above, and by combining this result with the known expressions for the invariants of a sextic in terms of the corresponding hyperelliptic $\vartheta$-zero values, he obtains a solution of the general equation of the sixth degree in terms of hyperelliptic $\vartheta$-zero values, analogous to Hermite's solution of the general quintic equation in terms of elliptic modular functions.

The determination of the complete system of invariant forms of the quaternary group of 51840 substitutions, $\uparrow$ which Maschke attacked next after his return to the gymnasium, was still more complex; but he succeeded also in this case by similar methods. Here he starts by determining the complete system of invariant forms for that subgroup which leaves the plane $z_{0}=0$ unchanged and which turns out to be the hessian group of 6.216 ternary substitutions. He thus solves incidentally an important problem of the theory of ternary groups.

Also for the quaternary group of $2 \cdot 7 ! / 2$ substitutions

* "La risoluzione della equazione di sesto grado," Rend. della R. Acc. dei Lincei, vol. 4, p. 181 (March, 1886). Another solution of the general sextic equation has been derived from Maschke's resolvent for the function $\Phi$ by Brioschi ; see the references in the Encyklopädie, I B $3 \mathrm{f}$, p. 550.

$\dagger$ " Aufstellung des vollen Formensystems einer quaternären Gruppe von 51840 linearen Substitutionen," Göttinger Nachrichten, 1888, p. 78, and Math. Annalen, vol. 33, p. 317 (June, 1888).

¥For still another ternary group or rather class of groups Maschke has determined the complete system of invariant forms, viz., for those so-called " monomial groups" which are generated by two substitutions of the form 
Maschke attempted to determine the complete system of invariant forms. He succeeded ${ }^{*}$ in solving, as a preliminary step, the problem for the subgroup of 2.168 quaternary substitutions isomorphic with Klein's ternary $G_{168}$, but the solution of the problem for the main group seems to have baffled his efforts. Still his occupation with this group led to an interesting geometrical investigation, the study of $a$ configuration of 140 straight lines $\dagger$ defined as follows : Let $x_{0}, x_{1}, \ldots, x_{6}$ be supernumerary line coordinates connected by the two relations

$$
\sum_{i=0}^{6} x_{i}=0, \sum_{i=0}^{6} x_{i}^{2}=0 ;
$$

then according to a general theorem of Klein's, every even permutation between the $x$ 's is equivalent to a collineation between the point coordinates, and the totality of these collineations constitute the group in question, viewed as a collineation group.

If these operations are applied to a line for which $x_{1}=x_{2}=x_{3}, x_{4}=x_{5}=x_{6}$, the line takes only 140 different positions, and this is the configuration which Maschke studies in detail. Among his results I will only mention that every line $L$ of the configuration is met by 36 other lines of the configuration; these 36 transversals intersect each other in hexads in 6 points of the line $L$ having a peculiar position which Maschke calls metharmonic; $\$$ they not only lie in three ways in involution, forming in proper notation the pairs $(1,4)(2,6)(3,5) ;(1,5)(2,4)(3,6) ;(1,6)(2,5)(3,4)$, but at the same time $(1,5)$ is harmonically divided by $(2,4)$; $(2,6)$ by $(3,5) ;(3,4)$ by $(1,6)$.

After the completion of the paper just mentioned, Maschke's scientific productivity suffered an interruption of several years

$$
\begin{array}{lll}
z_{1}^{\prime}=z_{2}, & z_{2}^{\prime}=z_{3}, & z_{3}^{\prime}=z_{1}, \\
z_{1}^{\prime}=a_{1} z_{1}, & z_{2}^{\prime}=a_{2} z_{2}, & z_{3}^{\prime}=a_{3} z_{3},
\end{array}
$$

see "On ternary substitution-groups of finite order which leave a triangle unchanged," Amer. Journal of Math. vol. 17, p. 168 (December, 1894).

* In 1888, published several years later in the Chicago Congress papers (1893), p. 175 under the title "The invariants of a group of 2.168 linear quaternary substitutions."

† "Ueber eine merkwürdige Configuration gerader Linien im Raum," Göttinger Nachrichten, 1889, p. 384, and Math. Annalen, vol. 36, p. 190 (July, 1889).

† Later on Maschke returned to such systems of metharmonic points in the paper "On systems of six points lying in three ways in involution" (Annals of Mathematics, vol. 10, p. 22, December, 1895), where he studies the properties of these point systems interpreted in the complex plane. 
through his branching off into electrotechnics. The next paper* of importance is dated November, 1895 ; it is an application of Cayley's representation of finite groups by color diagrams to the rotation groups of the regular bodies in three- and four-dimensional space. The results are remarkably simple and elegant; for instance, the color diagrams in space for the groups of the three-dimensional regular polyhedra are simply obtained by truncating the vertices, choosing the sides of the polygons thus introduced for black lines properly provided with arrows, and the remaining portions of the sides of the original polyhedron for red lines without arrows. The space diagrams thus constructed can be so projected into a plane that in the projection no two vectors intersect, a circumstance which makes these plane diagrams very perspicuous and useful for the study of the groups in question.

During the following years Maschke returned to the theory of finite groups of linear substitutions, but his papers have an essentially different character from his earlier investigations in the same field. He turns from the consideration of special groups to the discovery of general theorems on finite groups of linear substitutions, some of which have proved of the greatest importance in the further development of the theory.

The first of these theorems $\dagger$ states that every finite group of linear substitutions can be so transformed that in all its substitutions all the coefficients are cyclotomic, $i$. e., rationally expressible in terms of roots of unity, provided that the group contains at least one substitution whose multipliers are all different. The starting point for his proof is the theorem due to Jordan and Frobenius that for every linear substitution of finite period the multipliers are roots of unity, and he establishes his theorem step by step, first for the sum of the diagonal terms, then for the diagonal terms themselves, next for the terms of the first column, and finally for all the coefficients.

* "The representation of finite groups, especially of the rotation groups of the regular bodies of three- and four-dimensional space, by Cayley's color diagrams," Amer. Journal of Math., vol. 18, p. 156, and Göttinger Nachrichten, 1896, p. 1 (November, 1895).

$\dagger$ "Ueber den arithmetischen Charakter der Coefficienten der Substitutionen endlicher linearer Substitutionsgruppen," Math. Annalen, vol. 50, p. 492 (November, 1897). The paper is a sequel to the note "Die Reduction homogener Substitutionen von endlicher Periode auf ihre kanonische Form," Math. Annalen, vol. 50, p. 220 (March, 1897). Maschke's cyclotomic theorem has since been generalized in various directions by Burnside and Schur. 
Perhaps of even greater importance is the following theorem * to which Maschke was led in the course of the proof of his cyclotomic theorem : Every finite group of linear substitutions, all of whose substitutions contain in the same place (not in the principal diagonal) a coefficient equal to zero, is intransitive, i. e., it can be so transformed that the new variables fall into a number of sets such that the variables of each set are transformed among themselves. In Burnside's terminology, the essential part of the theorem may be briefly formulated as follows : Every yroup of linear substitutions of finite order is completely reducible.

In his proof Maschke makes use of the theorem which had been discovered shortly before by Loewy and Moore, that every finite group of linear substitutions leaves at least one definite hermitian form unchanged.

In the same year Maschke completed still another investigation on linear substitutions, viz., the determination of all ternary and quaternary collineation groups which are holoedrically isomorphic with the symmetric and alternating permutation groups. $\dagger$ His solution of this beautiful problem is based upon the theorem on hermitian forms just mentioned and upon a theorem due to Moore, giving the generational relations for the abstract groups holoedrically isomorphic with the symmetric and alternating groups. By a skilful application of these two theorems Maschke obtains with comparatively little computation the complete solution of the problem.

During the winter of 1900 , while he was giving a course on differential geometry, Maschke discovered a symbolic method for the treatment of differential quantics, and his scientific activity during the remaining years of his life was devoted to a detailed and systematic development of this discovery.

Maschke starts from the remark that if $F^{1}, F^{2}, \ldots, F^{n}$ are $n$ invariants of the quadratic differential quantic

$$
A=\sum a_{i k} d x_{i} d x_{l k}, \quad\left(a_{k i}=a_{i k}, i, k=1,2, \ldots, n\right),
$$

* See first reference in the last foot-note, where the theorem is proved under the same restricting assumption which is made in the cyclotomic theorem, and "Beweis des Satzes, dass diejenigen endlichen linearen Substitutionsgruppen, in welchen einige durchgehende Nullen vorkommen, intransitiv sind," Math. Annalen, vol. 52, p. 363 (December, 1898), where this restriction is dropped. Other proofs of the theorem have since been given by Frobenius, Burnside and schur, a generalization by Loewy.

$\dagger$ †"Bestimmung aller ternären und quaternären Collineationsgruppen, welche mit symmetrischen und alternierenden Buchstabenvertauschungsgruppen holoedrisch isomorph sind," Math. Annalen, vol. 51, p. 253 (November, 1897). 
then the expression

$$
\left(F^{1}, F^{2}, \ldots, F^{n}\right)=\left|a_{i k}\right|^{-\frac{1}{2}} \frac{\partial\left(F^{1}, F^{2}, \cdots, F^{n}\right)}{\partial\left(x_{1}, x_{2}, \cdots, x_{n}\right)}
$$

is also an invariant of $A$. By means of this theorem it is easy to build up invariants for those special differential quantics $A$ which are squares of complete differentials

$$
\begin{gathered}
\sum a_{i k} d x_{i} d x_{k}=(d f)^{2}=\left(f_{1} d x_{1}+\cdots+f_{n} d x_{n}\right)^{2}, \\
a_{i l_{k}}=f_{i} f_{l i}, \quad f_{i}=\frac{\partial f}{\partial x_{i}},
\end{gathered}
$$

and for systems of such quantics

$$
A^{1}=\left(d f^{1}\right)^{2}, A^{2}=\left(d f^{2}\right)^{2}, \cdots ;
$$

for instance*

$\left(f^{1}, f^{2}, \cdots, f^{n}\right)^{2}, \quad\left(u, f^{2}, \ldots, f^{n}\right)^{2}, \quad\left(u, f^{2}, \cdots, f^{n}\right)\left(v, f^{2}, \cdots, f^{n}\right)$,

etc., where $u, v$ are arbitrary functions of $x_{1}, x_{2}, \cdots, x_{n}$, are such invariants.

But every such invariant is at the same time an invariant for the general quantic $A$, provided it is homogeneous and of the second degree in the quantities $f_{i}^{1}$, in the quantities $f_{i}^{2}$, etc. Thus the expression $(d f)^{2}$ may be used as a symbol for the quadratic differential $A$ in the same sense in which $a_{x}^{n}$ is used as a symbol for the general binary $n$-ic in the ordinary theory of invariants, and $\left(d f^{1}\right)^{2},\left(d f^{2}\right)^{2}, \ldots$, etc., as equivalent symbols for $A$, if $A^{1}$, $A^{2}$, . . coincide. Thus for instance the above invariants, considered as symbolic expressions, have a real meaning for the general quantic $A$, viz., they are respectively equal to $n !$, the "first differential parameter" $\triangle_{1} u$, and the "intermediate differential parameter" $\nabla(u, v)$.

The remark that the total differential

$$
d F=F_{1} d x_{1}+\cdots+F_{n} d x_{n} \quad\left(=F_{x}\right)
$$

of an invariant $F$ is a covariant of $A$, leads to symbolic expressions for covariants; for instance the symbolic expression

$$
\left(f^{1}, f^{2}, \cdots, f^{n}\right)\left(u, f^{2}, \cdots, f^{n}\right)_{x} f_{x}^{1}
$$

represents a quadratic covariant.

\footnotetext{
* Maschke uses the more condensed notation $(f)^{2},(u f)^{2},(u f)(v f)$.
} 
A symbolic expression which contains higher derivatives of the symbolic function $f$ does not always have a real meaning, but only if these derivatives occur in certain combinations; the simplest combination of this kind, $f_{i} f_{k l}$, is equal to Christoffel's triple index symbol of the first kind

$$
f_{i} f_{k l}=\left[\begin{array}{c}
k l \\
i
\end{array}\right]
$$

Hence it follows, for instance, that the symbolic expression

$$
\left[\left(u, f^{2}, \cdots, f^{n}\right), f^{2}, \cdots, f^{n}\right]
$$

has a real meaning; it is equal to $(n-1)$ ! into the "second differential parameter" $\triangle_{2} u$.

Maschke first explained his method, in a preliminary way, for the special case $n=2$ in the paper "A new method for determining the differential parameters and invariants of quadratic differential quantics." *

In a later paper, "A symbolic treatment of the theory of invariants of quadratic differential quantics of $n$ variables," $\dagger$ Maschke gives an elaborate and systematic development of his method for the general case of $n$ variables. Very simple symbolic expressions are obtained for Christoffel's triple index symbol of the second kind, $\left\{\begin{array}{c}k l \\ i\end{array}\right\}$, his quadruple index symbol (ikrs), the quadrilinear covariant $G_{4}$, etc., and finally the three simplest general invariants (proper) of the second order are determined.

The great power of Maschke's symbolic method can best be seen from the applications which have been made to differential geometry in three-dimensional space by A. W. Smith in his doctor dissertation, $\$$ "The symbolic treatment of differential geometry," and to various problems of differential geometry in hyperspace by Maschke himself in his last two papers, "Differential parameters of the first order" $\S$ and "The KroneckerGaussian curvature of hyperspace." II

\footnotetext{
* Transactions of the American Mathematical Society, vol. 1, p. 197 (April, 1900).

†Ibid., vol. 7 (1907), p. 33.

$\ddagger$ Ibid., vol. 7. An interesting connection between Maschke's symbolic method and vector analysis has been established by L. Ingold in his doctor dissertation, "Vector interpretation of symbolic differential parameters."

\& Ibid., vol. 7, p. 69 (dated September, 1905).

\|l 1bid., vol. 7, p. 81 (dated September, 1905).
} 
In the first of these papers he determines all directions of a space $R_{\mu}$ of $\mu$ dimensions through a point $P$ of $R_{\mu}$, which are normal to all the directions of a second space $R_{\lambda}$ containing the point $P$, when $R_{\mu}$ and $R_{\lambda}$ are both immersed in a general space $R_{n}$ whose arc element is given by

$$
d s^{2}=\Sigma a_{i k} d x_{i} d x_{k} .
$$

In the second paper he develops the symbolic expression for Kronecker's extension of Gauss's curvature of a surface to a space of $n$ dimensions $R_{n}$, immersed in a euclidean space of $n+1$ dimensions, in terms of the symbols of the differential quantic

$$
d s^{2}=\Sigma \alpha_{i k} d u_{i} d u_{k} \quad(i, k=1,2, \cdots, n),
$$

which defines the arc element of $R_{n}$, and then generalizes his results to a space $R_{\lambda}$ of $\lambda$ dimensions immersed in a general space of $n$ dimensions, and defined by $n-\lambda$ relations

$$
U^{\prime}=\text { const }, \quad U^{\prime \prime}=\text { const, } \cdots, \quad U^{(n-\lambda)}=\text { const. }
$$

Further applications are sure to follow, and Maschke's symbolic method will doubtless play in the theory of differential quantics a rôle similar to that of the ordinary symbolic method of Aronhold and Clebsch in the theory of algebraic quantics, thus, together with his contributions to the theory of linear substitution groups, securing him a permanent place in the history of mathematics.

Freiburg, I. B.

Oskar Bolza.

August 8, 1908.

\section{NOTES.}

At the annual meeting of the American Mathematical SocIETY, to be held in the last week of December, President H. S. White will deliver his retiring address, the subject of which will be "Bezout's theory of resultants and its influence on geometry."

THE second regular meeting of the Southwestern Section of the American Mathematical Society will be held at the University of Kansas, Lawrence, Kansas, on Saturday, November 28. Titles and abstracts of papers to be presented at this meeting should be in the hands of the Secretary of the Section, Professor O. D. Kellogg, University of Missouri, Columbia, Mo., on or before November 14. 